\title{
Reclined trend of alkyl chain of sodium dodecylbenzenesulfonate molecules induced by friction
}

\author{
Xianzhang WANG ${ }^{1, \dagger}$, Yuan LIU ${ }^{1, \uparrow}$, Liran MA ${ }^{1, *}$, Xuefeng XU $\mathbf{X}^{2}$, Yu TIAN ${ }^{1}$ \\ ${ }^{1}$ State Key Laboratory of Tribology, Tsinghua University, Beijing 100084, China \\ ${ }^{2}$ School of Technology, Beijing Forestry University, Beijing 100083, China \\ Received: 01 March 2021 / Revised: 22 June 2021 / Accepted: 09 July 2021 \\ (C) The author(s) 2021.
}

\begin{abstract}
Surfactants tend to adsorb on the surface/interface mostly in a directional manner. The alkyl chain orientation and conformation order for molecular monolayers of sodium dodecylbenzenesulfonate (SDBS) at low concentrations are studied by using the sum frequency generation vibrational spectroscopy (SFG-VS). The molecular arrangement of the surfactants adsorbed at the solid/liquid interface is further investigated. It is found that the arrangement of the SDBS at the interface becomes relatively ordered with increasing bulk concentration. Meanwhile, the orientation angle reduces gradually, and the molecules tend to be upright state. In addition, the effect of friction on the conformation order and orientation angle are also analyzed. The intensity of the SDBS vibrational contraction peak becomes lower after friction, which indicates that the anion has a reorientation process at the interface. The arrangement of molecules becomes more disordered due to friction. The orientation angle increases slightly, which indicates the monolayer has an inclined trend relative to the lateral direction on the interface. A modified adsorption model considering friction effect is proposed. This work may provide a reference for the further study of adsorption mechanism and application of surfactants.
\end{abstract}

Keywords: friction; lubricating film; sum frequency generation (SFG); orientation angle; conformation order

\section{Introduction}

Surfactants are interfacially active compounds with amphiphilic molecules. Generally, surfactants consist of a charged headgroup and an extended hydrophobic alkyl chain [1]. One of the characteristics is that they tend to adsorb on the surface/interface in a directional manner. The adsorption behavior at the interface is determined by multiple forces including electrostatic force, hydrophobic interaction force, hydrogen bonding, van der Waals force, and other forces. The critical micelle concentration (CMC) of a surfactant is defined as the concentration of surfactant molecules that begin to self-aggregate to form micelles when the concentration reaches a certain level. Since micelles of surfactants can provide colloidal stability, corrosion inhibition, and boundary lubrication at solid/liquid interfaces, the surfactants are widely used in many fields.

In order to reveal the mechanisms of surfactant molecules adsorbed on solid surfaces, numerous studies have been taken on various surfaces at ultra-smooth surfaces such as: gold [2, 3], mica [4, 5], graphite [6, 7], C18 [8], single-walled carbon nanotubes (SWCNTs) $[9,10]$, and multi-walled carbon nanotubes (MWCNTs) [11], or macro scale surface such as filter paper [12, 13], silica [14, 15], silica gel [16], $\mathrm{CaF}_{2}$ [17], alumina [18, 19], alumina particle, carbonate materials [20, 21], and stainless steel [22]. In these studies, various detection methods such as atomic force microscopy (AFM), scanning probe microscopy (SPM), dual polarization interferometry (DPI), quartz crystal microbalance

$\uparrow$ Xianzhang WANG and Yuan LIU contributed equally to this work.

* Corresponding author: Liran MA, E-mail: maliran@mail.tsinghua.edu.cn 
(QCM), scanning UV/visible spectrophotometer, X-ray diffraction (XRD), X-ray photoelectron spectroscopy (XPS), Raman spectroscopy, and sum frequency generation (SFG) were used. Since sum frequency generation vibrational spectroscopy (SFG-VS) has natural sensitive interface selectivity, it can be used to quantitatively detect the absorption with various vibrational mode, furthermore to determine the orientation, arrangement, and conformational change of the molecules at the interfaces [23]. Tyrode et al. [15] studied the adsorption of a cationic surfactant cetyltrimethylammonium bromide $\left(\mathrm{C}_{16} \mathrm{TAB}\right)$ on hydrophilic silica. They observed a continuous spectrum, which is considered as the result of the combination interaction of headgroup binding and hydrophobic association. Takeshita et al. [17] applied the heterodyne-detected total internal reflection SFG spectroscopy to $\mathrm{CaF}_{2} /$ liquid interfaces and obtained the polar orientations of the water molecules and sodium dodecyl sulfate (SDS). Das et al. [9] investigated the degree of interfacial ordering for SDS surfactants adsorbed on the surface of SWCNT and the effect of bulk concentration of surfactant solution on alkyl chain order. Algoul et al. [10] investigated the interfacial ordering of SDS within solution-processed thin films under various environmental conditions. Both of found that the interfacial conformational order of SDS decreases with decreasing concentration, which results in the adsorption of surfactants on the nanotube surfaces more disordered $[9,10]$.

Tribology has been developed greatly in various fields in recent years. Meng et al. [24] reviewed the recent advances in research pertaining to different aspects of tribology including lubrication, wear and surface engineering, biotribology, high temperature tribology, and computational tribology. Ghaednia et al. [25] reviewed the models of elastic-plastic contacts over a wide variety of geometries and loading situations. Ma and Luo [26] systematically reviewed the significant progress of thin film lubrication (TFL) in the past two decades. Later, a series of review studies on molecular behaviors in TFL were carried out from the different perspectives: film formation [27], direct observation [28], and superlubricity [29]. Ta et al. [30] investigated the impact of chosen force fields and applied load on thin film. Ren et al. [31] compared the tribological properties of the composite coatings with different matrices and fillers, and analyzed the lubrication mechanisms. Martín-Alfonso et al. [32] studied the effect of the thickener concentration on the rheological, chemical, thermal, tribological properties, and AFM microstructure of these systems for montmorillonite/ castor oil. Zhang et al. [33] analyzed the mixed lubrication of a thrust bearing with fractal rough surfaces. Surfactants can be used as lubricants to improve the lubrication state of friction interface, reduce friction, and wear. Li et al. [34] found that an extremely low friction coefficient can be achieved on silica surface and graphitic surface [35] lubricated by $\mathrm{C}_{16} \mathrm{TAB}$ aqueous solution. He et al. [36] studied the frictional behaviors of SDS surfactant at surfaces of charged stainless steel under different electrode potentials. They presented a four-stage adsorption model at the solid/liquid interface. Later, Zhang et al. [37] investigated the frictional behaviors of stainless steel in SDS aqueous solution, and found that the boundary lubrication behaviors are not only related to the mass and structure of the adsorbed SDS boundary film, but also affected by the driving speed and load.

SFG-VS as a second-order nonlinear optical process, is extremely sensitive to interfacial molecules. It can be used to investigate the underlying microscopic mechanisms, which are related to the molecular arrangement at interfaces. For a symmetrical object, the electric dipole is approximately zero, and the second-order polarization rate will not generate a SFG signal. On the contrary, the bulk phases of the substances on both sides of the surface/interface are different. Since the symmetry of the molecule is broken, the second-order nonlinear response may not be zero, the SFG signals at surface/interface can be generated. SFG-VS can selectively detect the properties at the interface without the influence of bulk phase. SFG realizes the measurement of the orientation angle of the molecular group interface by measuring the intensity ratio of the group vibration spectra in different polarization directions. Compared with linear spectroscopies, more combinations of incident and emergent polarization can be obtained, so the resulting orientation angle is more accurate. Lis et al. [38] investigated the influence of the motion of liquid flows along a solid surface on interfacial chemistry by using SFG-VS. They found that the flow could cause a reversible change in surface charge, and then rearrange 
the water molecules on the surface of calcium fluoride $\left(\mathrm{CaF}_{2}\right)$ and fused silica. Gao et al. [39] revealed the interaction between water and phosphoric acid molecules at solution $/ \mathrm{SiO}_{2}$ interface in the process of superlubricity. Wang et al. [40] studied the molecular chemical structure on poly methyl methacrylate (PMMA) surface, and they deduced the ranges and distribution of orientation of the ester methyl group at the PMMA/air interface.

In most of the existing models, the molecules of anionic surfactants were adsorbed on hydrophilic surface as monomers at low concentrations. Meanwhile, the headgroups somewhat tilt upwards due to the electrostatic repulsion. However, the exact value of tilt angle is still unclear. How much the tile angle changes due to concentration change and friction still needs exploring. The aim of this work is to investigate the conformation and orientation of an anionic surfactant sodium dodecylbenzenesulfonate (SDBS) and the effects of concentration and friction on them. The results might be beneficial to change frictional characteristics by controlling the molecular arrangement of surfactants.

\section{Experimental}

\subsection{Preparation of samples}

An anionic surfactant sodium dodecylbenzenesulfonate (> 99\%) whose chemical formula is $\mathrm{C}_{18} \mathrm{H}_{29} \mathrm{NaO}_{3} \mathrm{~S}$, is a commercial product. The ultrapure water with the electrical resistance of $18.2 \mathrm{M} \Omega$ was used for preparing SDBS aqueous solutions, the concentration range of which was very wide $(0.01-2 \mathrm{mM})$. The lower disk specimen used was fused silica, and the roughness of which was around $20 \mathrm{~nm}$. The upper pin specimen was homemade with polytetrafluoroethylene (PTFE) material. Its diameter was $5 \mathrm{~mm}$, and the resulting contact area was $19.635 \mathrm{~mm}^{2}$. Since SFG is sensitive to monolayer, fused silica samples need deep cleaning before use in related experiments. The fused silica and PTFE samples were cleaned thoroughly in the following method: The samples were firstly washed by the ultrasonic cleaning oscillation in acetone and ethanol for 15 mins; after this, they were washed with plenty of ultrapure water, and then dried with pure nitrogen. The specific preparation method for the experiments was: The quartz samples were firstly immersed in the aqueous SDBS solution for about 15 mins; then placed vertically and remove the thick liquid layer of the samples by using clean tissue. The samples with thin liquid layer were then ready for the tests. All the surfaces of fused silica were some slight negatively charged after these treatments. Because the $\mathrm{pH}$ value of solution is greater than the point of zero charge (pzc) of $\mathrm{SiO}_{2}$. The dissolution reaction is dominated by the hydrolysis of the $\mathrm{Si}-\mathrm{O}-\mathrm{Si}$ bond [38].

$$
\equiv \mathrm{Si}-\mathrm{O}-\mathrm{Si}(\mathrm{OH})_{2} \mathrm{O}^{-}+\mathrm{H}_{2} \mathrm{O} \rightleftharpoons \mathrm{Si}-\mathrm{OH}+\mathrm{Si}(\mathrm{OH})_{3} \mathrm{O}^{-}
$$

\subsection{SFG spectroscopy}

The experimental setup is as following: An ultrafast sapphire laser system (Mai Tai SP) emits a beam with 100 femtosecond pulses, then it turned to be a beam with energy of $5 \mathrm{~mJ}$, wavelength of $800 \mathrm{~nm}$, frequency of $1 \mathrm{kHz}$, and full width half maximum (FWHM) of $13 \mathrm{~nm}$, through a high-power pump laser (Empower) and a flagship amplifier system (Spitfire); after that, the beam was split in three: the first one was for Infrared Ray (IR) pulse generation, the second one was for visible pulse shaping, and the other beam was used for optional stimulated Raman experiment rather than SFG. The pulse energies of visible and IR beams were both $2 \mathrm{~mJ}$. The visible beam had a wavelength of $800 \mathrm{~nm}$ and a broadband IR beam that was centered at $3,448 \mathrm{~nm}$ with a FWHM of $200 \mathrm{~cm}^{-1}$. In the SFG experiments, the visible beam and the tunable IR beam were overlapped spatially and temporally at the solution/substrate interface. The incident angles on the sample were $\beta_{\text {vis }}=50^{\circ}$ and $\beta_{\mathrm{IR}}=30^{\circ}$ for the visible and IR beams, respectively. Through a series of optical components, the two beams eventually converge on the surface of the sample. The fused silica sample was mounted on a sample stage holder, which has six degrees of freedom: translation in three coordinates and rotation about three coordinate axes. Two polarizers were placed in the IR and SFG light paths. The reflected SFG spectra were recorded with an electron-multiplied charge-coupled device (EMCCD) camera (Newton, Andor Technologies, USA), connected to a spectrometer (Horiba) (Fig. 1(a)). The SFG intensity is then obtained by integrating the area under the SFG spectrum. All spectra were collected under the 
(a)

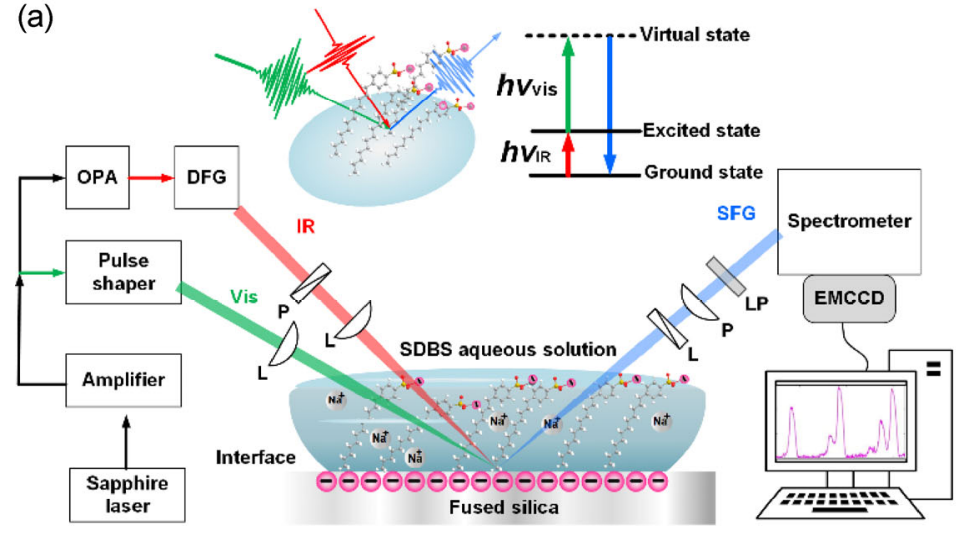

(c)

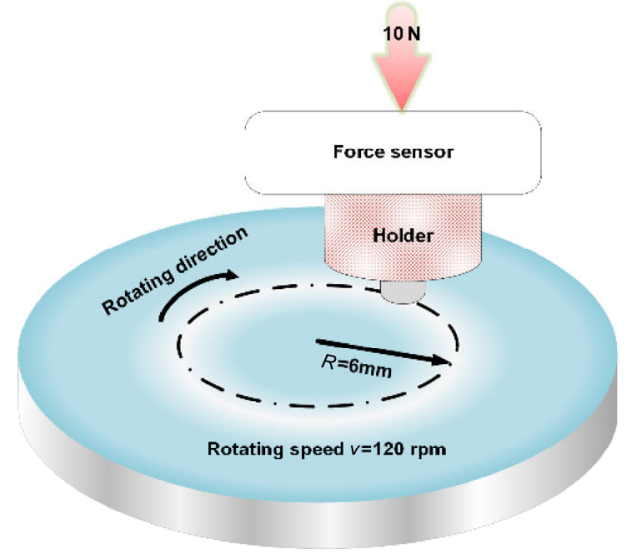

(b)

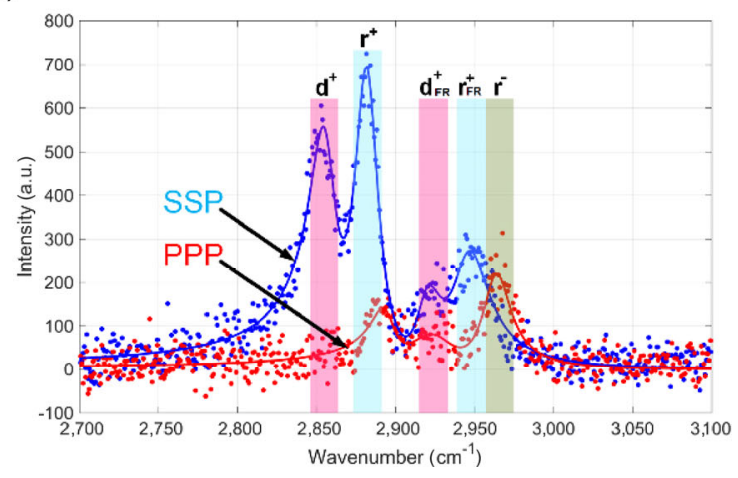

(d)

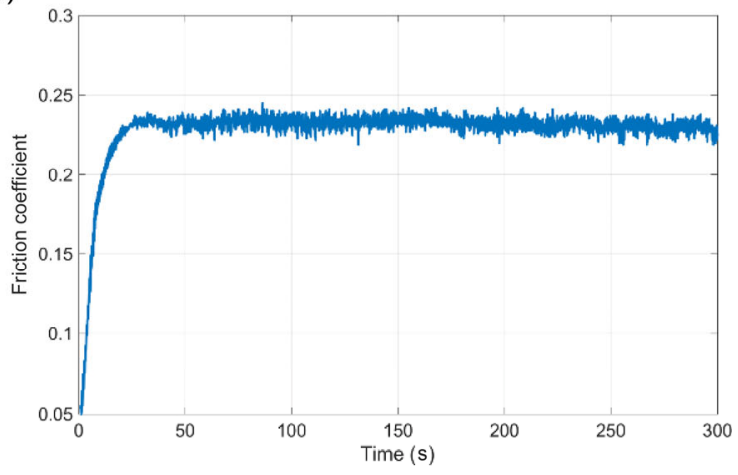

Fig. 1 (a) Schematic of experimental setup for probing the structure of SDBS monolayer adsorbed on the fused silica interface and its conformation. The polarized IR beam and the pulse-shaped visible beam are focused at the liquid/solid interface to produce the SFG signal. L, P, and LP present focusing lens, polarization, and low pass filter, respectively. The SFG beam is directed to a spectrometer and detected with an EMCCD camera. The upper diagram shows the SFG energy level transition, the dotted line represents a virtual level of the Raman transition; (b) SFG spectra of SDBS in the CH stretching region under polarizations of SSP and PPP; (c) schematic illustration of the pin-on-disc surface contact for the frictional tests; and (d) the friction coefficient during a frictional test.

SSP (S-polarized sum frequency, S-polarized visible, P-polarized infrared) or PPP (P-polarized sum frequency, P-polarized visible, P-polarized infrared) polarization conditions. The schematic of energy level transition is plotted on the left top of Fig. 1(a). The SFG is a combined process of infrared vibration excitation and an anti-Stokes Raman emission.

Molecular vibration is mainly divided into two categories: stretching vibration and bending vibration. Generally, the stretching vibration is caused by a change in bond length, and is divided into symmetric vibration and asymmetric vibration. Fermi resonance occurs because the energy is transferred from one vibration mode to another, resulting in an exchange and transfer of energy. When the multiple or group frequency of one vibration is close to the fundamental frequency of another vibration, the interaction occurs to produce a strong absorption peak or splitting peak. Two general SFG spectra recorded in the $\mathrm{CH}$-stretching region under SSP polarization and PPP polarization are shown in Fig. 1(b). The SFG spectra of SDBS on a fused silica surface exhibit a pronounced four peak structure under SSP polarization, while three main peak structures under PPP polarization. Under SSP polarization, the two major peaks at $\sim 2,850$ and $2,880 \mathrm{~cm}^{-1}$ can be assigned to the $\mathrm{CH}_{2}$ and $\mathrm{CH}_{3}$ symmetric stretching vibrations $\left(\mathrm{CH}_{2}\right.$,ss and $\left.\mathrm{CH}_{3}, \mathrm{ss}\right)$, which are marked as $d^{+}$and $r^{+}$, respectively. Their Fermi resonances, $\mathrm{CH}_{2}-\mathrm{FR}$ and $\mathrm{CH}_{3}$-FR (marked as $d_{\mathrm{FR}}^{+}$and $r_{\mathrm{FR}}^{+}$), were also observed at 2,925 and 2,945 $\mathrm{cm}^{-1}$, respectively. Under PPP polarization, the asymmetric stretching band at $\sim 2,968 \mathrm{~cm}^{-1}$ is assigned to the $\mathrm{CH}_{3}$ asymmetric stretching vibration (marked as $r^{-}$), which is more clearly visible. The other two peaks are $\mathrm{CH}_{3}$ symmetric 
stretching vibration $\left(\mathrm{CH}_{3}, \mathrm{ss}\right)$ and $\mathrm{CH}_{2}$ Fermi resonances $\left(\mathrm{CH}_{2}-\mathrm{FR}\right)$. These assignments were consistent with those of previous SFG-VS studies [9, 10, 41-44]. As discussed in the supporting information, in order to revise the IR pulse shape and normalization, we first measured the SFG signal of the sample, and then measured the signal of gallium arsenide (GaAs) in the same state. The final sum frequency generation signal was the ratio of the intensity between two successive measurements. In the current study, all the sample spectra were normalized against the SFG signals from the surface of $\mathrm{GaAs}$, the general spectra of which are plotted in Fig. S1 in the Electronic Supplementary Material (ESM)

\subsection{Tribological test}

The tribological tests were carried out by using the universal mechanical tester (UMT, Bruker, USA). The homemade PTFE cylinders with diameter of $5 \mathrm{~mm}$ was used as upper samples. The lower samples were the fused silica with diameter of $25.4 \mathrm{~mm}$ and thickness of $5 \mathrm{~mm}$. In the experiments, the fused silica plates were attached to a rotating platform along the motor, and the PTFE samples were fixed on a holder, which is connected to the force sensor (DFH 5.0). The applied force was $10 \mathrm{~N}$, the rotating radius was $6 \mathrm{~mm}$, and the velocity was $120 \mathrm{rpm}(0.075 \mathrm{~m} / \mathrm{s})$, as shown in Fig. 1(c). The experimental procedure was designed as following: Firstly, the SFG measurements were performed on the prepared samples; they were then taken to do the tribological tests by using UMT immediately; the tests lasted at least five minutes until the friction coefficient reaches a steady state, as shown in Fig. 1(d); at last, the samples were taken back to do the SFG measurements immediately. The time of transportation must be controlled strictly, in order to avoid the possible changes in the status of samples. All the tests were performed at room temperature of $23^{\circ} \pm 0.5^{\circ}$ in ambient air.

\section{Results and discussion}

\subsection{Friction leads to the changes of conformation order and tilt angle}

Based on the changes of each peak under the two polarizations of SSP and PPP, we can preliminarily determine the adsorption tendency of SDBS anion on the fused silica and the effect of friction on conformation.
Generally, the intensity of Fermi resonance is weaker than that of corresponding stretching peaks under SSP polarization. However, the symmetric stretching peaks $\left(d^{+}\right.$and $\left.r^{+}\right)$are lower than the peaks caused by Fermi resonance $\left(d_{\mathrm{FR}}^{+}\right.$and $\left.r_{\mathrm{FR}}^{+}\right)$when the frictional tests were carried out in this study. SFG spectra of an SDBS monolayer on fused silica surface in the $\mathrm{CH}$ stretching region $\left(2,820-3,000 \mathrm{~cm}^{-1}\right)$ with various concentrations are plotted in Figs. 2(a)-2(d). The solid lines are the results of fitting the measured data by Lorentzian line shapes, Eq. (S2) in the ESM, to guide the eye. As can be seen from Figs. 2(a) and 2(c), for both cases with concentration of 0.1 and $1 \mathrm{mM}$, the amplitudes of $\mathrm{CH}_{2}$ symmetric stretching band $d^{+}$are greater than that of $\mathrm{CH}_{2}$ Fermi resonances band $d_{\mathrm{FR}}^{+}$, and the amplitudes of symmetric stretching $\mathrm{CH}_{3}$ band $r^{+}$are greater than that of Fermi resonances $\mathrm{CH}_{3}$ band $r_{\mathrm{FR}}^{+}$. After friction, the amplitudes of $d^{+}$become less than that of $d_{\mathrm{FR}}^{+}$and the amplitudes of $r^{+}$are greater than that of $r_{\mathrm{FR}}^{+}$. In addition, a significant decrease in SFG intensity after friction was observed. It was shown that significant changes of amplitude of the $d^{+}$and $r^{+}$bands, while the $d_{\mathrm{FR}}^{+}$and $r_{\mathrm{FR}}^{+}$have a negligible change under SSP polarization. Compared with the Fermi resonances of $\mathrm{CH}_{2}$ and $\mathrm{CH}_{3}$ group $d_{\mathrm{FR}}^{+}$and $r_{\mathrm{FR}}^{+}$, the symmetric stretching bands $\left(d^{+}\right.$and $\left.r^{+}\right)$become weaker due to friction. As can be seen from Figs. 2(b) and 2(d), under PPP polarization, the intensities turn weaker overall. The peaks of $\mathrm{CH}_{3}$ symmetric stretching band $r^{+}$and Fermi resonances of $\mathrm{CH}_{2}$ band $d_{\mathrm{FR}}^{+}$are nearly disappeared, while the amplitude of asymmetric stretching band $r^{-}$decreases but remains apparent.

To understand the conformation of surfactant molecules, it is necessary to consider the local skeletal structure of the $\mathrm{CH}_{2}$ group. For the all-trans conformation, the contribution of $\mathrm{CH}_{2}$ group nearly canceled by one another due to its centrosymmetric distribution. Hence, the terminal $\mathrm{CH}_{3}$ are the only ones that have contribution. When the Gauche defect is present, the local symmetry is broken. Hence, it would response and generate the SFG signal [9]. The schematic is shown in Fig. 2(e). The amplitude of $\mathrm{CH}_{2}$ symmetric stretching band $d^{+}$presents the number of Gauche defects build-up of methylene groups in all-trans configurations. A weaker $d^{+}$indicates that the alkyl chain has a trans structure, and the order of the alkyl chain is better. Otherwise, if $d^{+}$is stronger, the alkyl 
chain has a stronger Gauche defect, and the arrangement of the alkyl chain at the interface is relatively disordered. The ratio of amplitude of $\mathrm{CH}_{3}$ symmetric stretching band $r^{+}$to that of $\mathrm{CH}_{2}$ symmetric stretching band $d^{+}$, $A_{\mathrm{CH}_{3}, \mathrm{sS}} / A_{\mathrm{CH}_{2}, \mathrm{ss}}$, is usually used to indict the conformational order of the alkyl chain [9, 41, 42, 45-47].
This is directly related to the rise in conformation order and surface density of $\mathrm{CH}_{3}$ groups. The alkyl chain becomes conformationally more ordered due to the increase of surface density when this ratio increases.

However, the value of $A_{\mathrm{CH}_{3}, \mathrm{ss}} / A_{\mathrm{CH}_{2}, \mathrm{ss}}$ does not remain constant, when optimizing the parameters

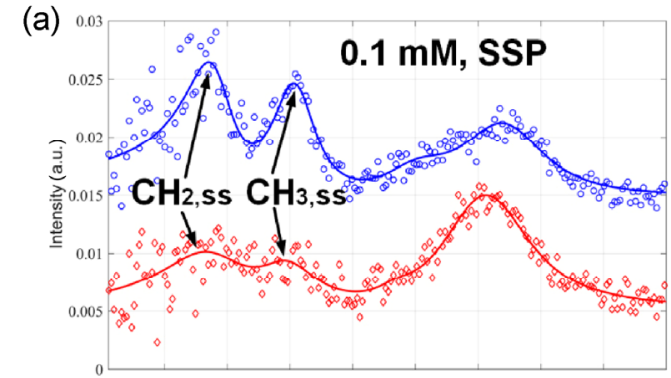

(c)

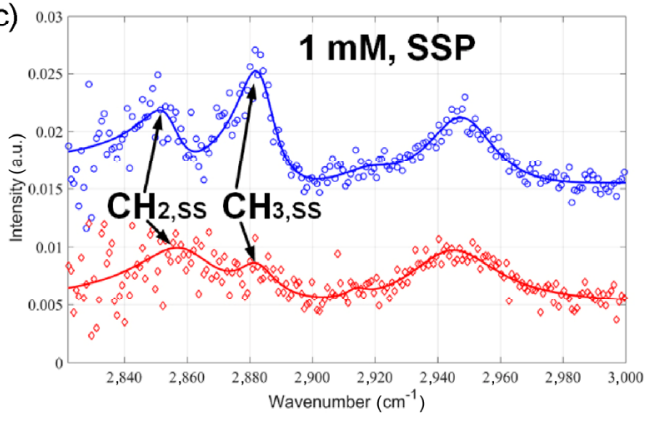

(e)

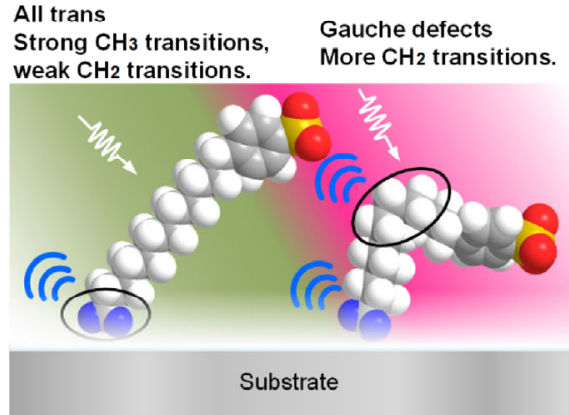

(g)

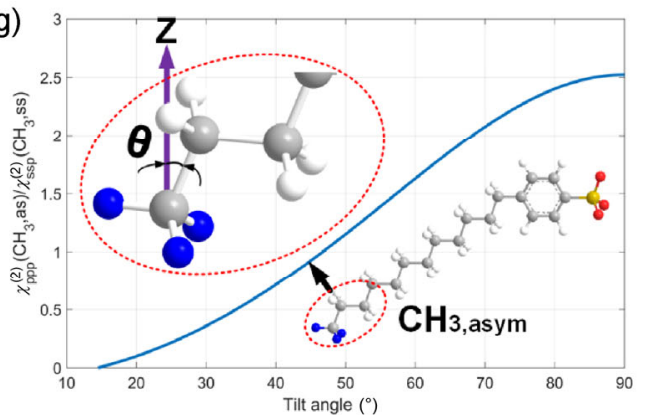

(b)

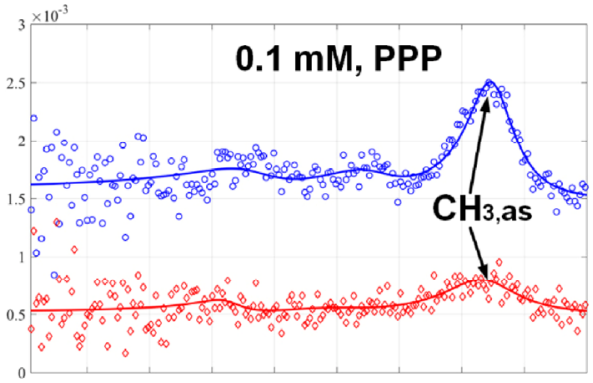

(d)

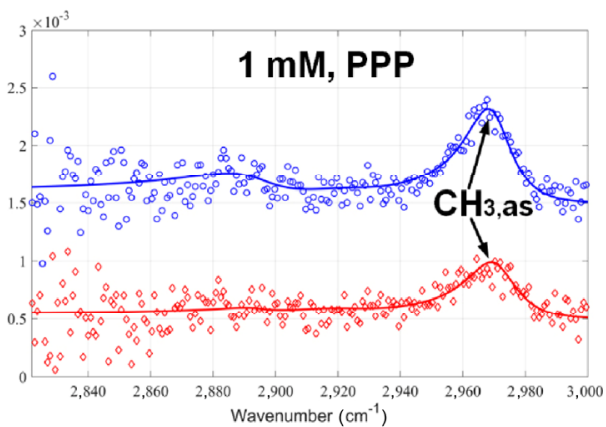

(f)

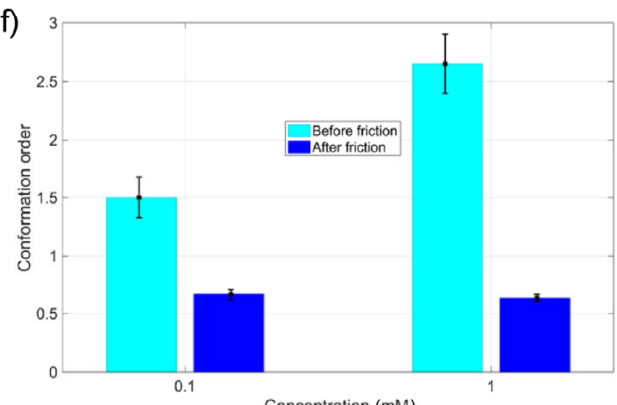

(h)

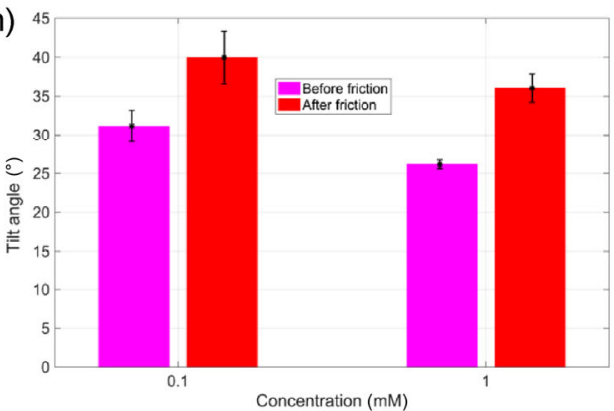

Fig. 2 (a-d) SFG spectra with various concentrations and polarizations before and after friction, which were offset vertically for clarity. The blue and red lines present the spectra before and after friction, respectively. The concentrations and polarization combinations are: (a) $0.1 \mathrm{mM}$, SSP; (b) $0.1 \mathrm{mM}$, PPP; (c) $1 \mathrm{mM}$, SSP; and (d) $1 \mathrm{mM}$, PPP polarization. (e) Schematic of the SDBS in an all-trans $\mathrm{CH}_{2}$ (left) and contribution from a gauche defect (right); (f) the conformation orders before and after friction with concentrations of 0.1 and $1 \mathrm{mM}$; $(\mathrm{g})$ the ratio $\chi_{\mathrm{ppp}}^{(2)}\left(\mathrm{CH}_{3}\right.$, as $) / \chi_{\mathrm{ssp}}^{(2)}\left(\mathrm{CH}_{3}, \mathrm{ss}\right)$ for the vibrational modes of the terminal methyl group as a function of its tilt angle, $\theta$; and $(\mathrm{h})$ the tilt angles before and after friction with concentrations of 0.1 and $1 \mathrm{mM}$. 
in the curve fitting process. Instead, the value of $\left(A_{\mathrm{CH}_{3}, \mathrm{ss}} / \Gamma_{\mathrm{CH}_{3}, \mathrm{ss}}\right) /\left(A_{\mathrm{CH}_{2}, \mathrm{ss}} / \Gamma_{\mathrm{CH}_{2}, \mathrm{ss}}\right)$ keeps constant, and the value just has a slight change from each other, which were demonstrated in Fig. S3 in the ESM. Consequently, $\left(A_{\mathrm{CH}_{3}, \mathrm{ss}} / \Gamma_{\mathrm{CH}_{3}, \mathrm{ss}}\right) /\left(A_{\mathrm{CH}_{2}, \mathrm{ss}} / \Gamma_{\mathrm{CH}_{2}, \mathrm{ss}}\right)$ is used as the conformational order indicator for the SDBS monolayer on fused silica in this study. This is also a correcting process to reduce the error of curve fitting of the qualitative measurements. The conformation order is plotted in Fig. 2(f). As can be seen from Fig. 2(f), the molecular arrangement becomes relative disordered after friction. The friction bends the alkyl chain and generate more Gauche defects in the structure, which could collectively contribute to the vibrational response of $\mathrm{CH}_{2}$ group. Thus, the $\mathrm{CH}_{2}$ stretching bands appear in the SFG spectra. This could be also because these alkyl chains of molecules twine around each other to form messy structures.

The tilt angle is determined by the orientation of its terminal methyl group, which is relative to the interface normal (as shown in Fig. 2(g)). The ratio $\chi_{\mathrm{ppp}}^{(2)}\left(\mathrm{CH}_{3}, \mathrm{as}\right) / \chi_{\mathrm{ssp}}^{(2)}\left(\mathrm{CH}_{3}, \mathrm{ss}\right)$, which is independent of the incident light intensity, can be expressed as a function of tilt angle of the methyl group, see Eq. (S11) in the ESM. It is also can be given by

$$
\frac{A_{\mathrm{CH}_{3}, \text { as }} / \Gamma_{\mathrm{CH}_{3}, \text { as }}}{A_{\mathrm{CH}_{3}, \mathrm{ss}} / \Gamma_{\mathrm{CH}_{3}, \mathrm{ss}}}=\frac{15.44 \cos \theta-16.26 \cos ^{3} \theta}{4.4 \cos \theta-2.4 \cos ^{3} \theta}
$$

Note that the orientation angle, $\theta$ is assumed consistent with delta distribution, which is a single average value. The term of the left-hand side of Eq. (1) was calculated from the SGF experimental data, whose spectra were shown in Figs. 3(a) and 3(b). The fitting parameters $\left(A_{\mathrm{as}}, \Gamma_{\mathrm{as}}, A_{\mathrm{ss}}\right.$, and $\left.\Gamma_{\mathrm{ss}}\right)$ were obtained by using Eq. (S2) in the ESM. So, the tilt angle can be calculated. Friction provides a reorientation of the alkyl chain, with the angle becoming larger and tending to recline or lie down on the interface. Then, the tilt angles for both cases with various SDBS concentrations are plotted in Fig. 2(h). The friction can increase the orientation angle, that is the SDBS molecule has a "lay down" trend due to friction. There are some possible potential reasons: 1) It might be dictated by the deflection of the long polymer chain molecular based on polymer physics. Friction can make the alkyl chains of SDBS bent, twisted and even twine together. 2) The charges on the silica are then neutralized by the opposite charge, which are created by friction against a PTFE cylinder. As a result, the electrostatic repulsion force becomes smaller, resulting in a change in the orientation angle. 3) Due to friction, the gap of the molecular chains becomes larger, which leads to a smaller repulsive force between the molecular chains and a larger orientation angle. 4) The changes of molecular interactions at interface such as van der Waals force due to friction. It is very likely that a combination of several of these are involved. However, the clear underlying mechanisms remain to be further explored.

\subsection{Effect of SDBS concentration}

SFG spectra of an SDBS monolayer on fused silica surface in the $\mathrm{CH}$ stretching region with various concentrations are plotted in Figs. 3(a) and 3(b). Likewise, the solid lines are the results of fitting the measured data by Lorentzian line shapes (see Eq. (S2) in the ESM) to guide the eye. These SFG spectra exhibit a pronounced four peak structures $\left(d^{+}, r^{+}, d_{F R}^{+}\right.$, and $\left.r_{F R}^{+}\right)$ under the SSP polarization, while three peak $\left(r^{+}, d_{F R}^{+}\right.$, and $r^{-}$) structures under the PPP polarization, in which $r^{-}$band is more clearly visible. The increase of concentration results in a strong increase in the symmetric stretching band of $\mathrm{CH}_{3}$ group $r^{+}$, which is caused by the rise in conformation order and surface density of methyl groups. From Fig. 3(a), compared with $0.1 \mathrm{mM}$, the methyl vibration peak with the concentration of $1 \mathrm{mM}$ exhibits a red shift in their optical absorption spectra, this is agreed well with the conclusion in Ref. [48]. In conclusion, as the concentration increases, the tile angle decreases, and the alkyl chain becomes relatively straight.

First, the conformation order versus SDBS concentration is plotted in Fig. 3(c). As discussed previously, all the parameters $\left(A_{\mathrm{CH}_{2}}, A_{\mathrm{CH}_{3}}, \Gamma_{\mathrm{CH}_{2}}, \Gamma_{\mathrm{CH}_{3}}\right)$ are obtained from the SSP spectra in Figs. 3(a) and 3(b). It shows a clear trend that the conformational order increases with increasing SDBS bulk concentration. This means $\mathrm{CH}_{2}$ contributions to the SFG spectra eventually decrease, while $\mathrm{CH}_{3}$ contributions increase. The number of $\mathrm{CH}_{2}$ in the trans arrangement increases, 
(a)

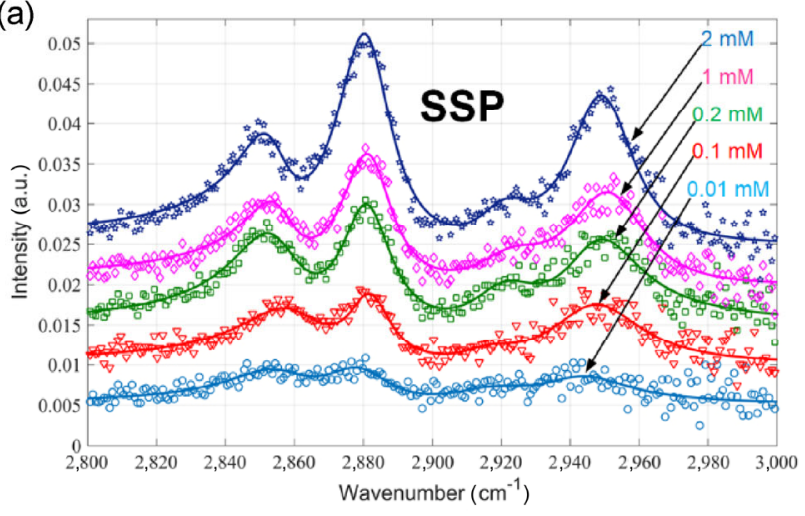

(c)

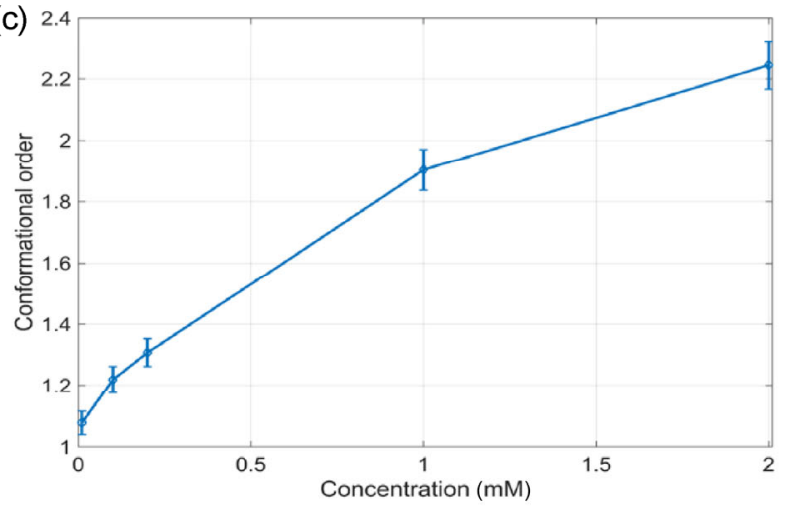

(b)

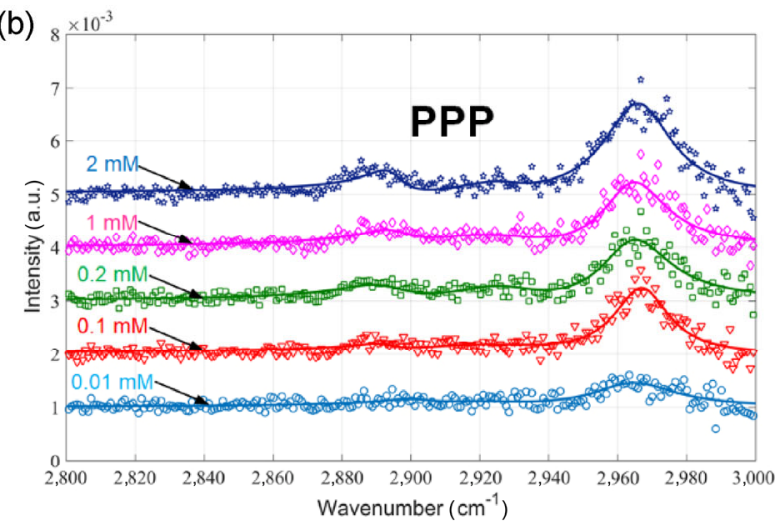

(d)

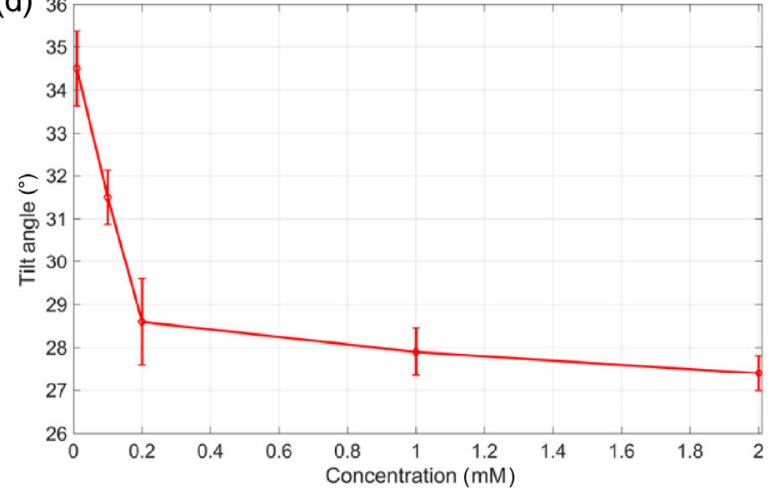

Fig. 3 (a, b) SFG spectra of SDBS monolayer on fused silica in the CH stretching region with various concentrations: (a) under SSP polarization and (b) under PPP polarization. The smooth solid lines correspond to the fits using Eq. (S2) in the ESM and the spectra were offset vertically for clarity: (c) conformation order indicator as a function of the SDBS concentration in this study and (d) orientation angle as a function of the SDBS concentration.

which makes the arrangement of molecules at the interface more regular and ordered. The main reason might be that the adsorption of SDBS on interface increases, and the chain-to-chain interaction leads to a decrease in Gauche defect.

Then, the molecular orientation is analyzed. The tilt angle with various SDBS concentration is plotted in Fig. 3(d). As can be seen from Fig. 3(d), the tilt angle, which is between the methyl axis and surface normal, is around $30^{\circ}$ when the SDBS concentration is $0.01 \mathrm{mM}$. The tilt angle decreases sharply with increasing concentration. As the concentration further decreases the tilt angle slowly approaches a constant value that is about $27^{\circ}$.

\subsection{An adsorption model for anionic surfactant considering friction effect}

The adsorption characteristics of SDS on negatively charged stainless steel surface were investigated by Zhang et al. [22]. They found that the mass of the adsorbed SDS molecules increases with increasing bulk concentration. Meanwhile, the structure of the adsorbed layer was changed to hemimicelles from monomers. The adsorption isotherm for the four-stage model is plotted in Fig. 4(a). This model just presented the phenomenon that the ionic surfactant monomers stand at an angle to the surface. However, the degree of the tilt angle is still unclear, and no direct evidences are provided to identify the underlying mechanisms. Based on the idea of four regime models, a modified adsorption model considering friction effect for the anionic surfactant at low concentrations is proposed. The schematic representation of the model is plotted in Fig. 4(b). According to the micellization of SDBS molecules, it follows a four-stage adsorption process: 1) At very low bulk concentrations, the molecules are adsorbed on the surface of the substrate as monomers. The headgroups tilt upwards somewhat due to the electrostatic and hydrophobic interactions between anionic surfactant molecules and substrate surface 
(a)

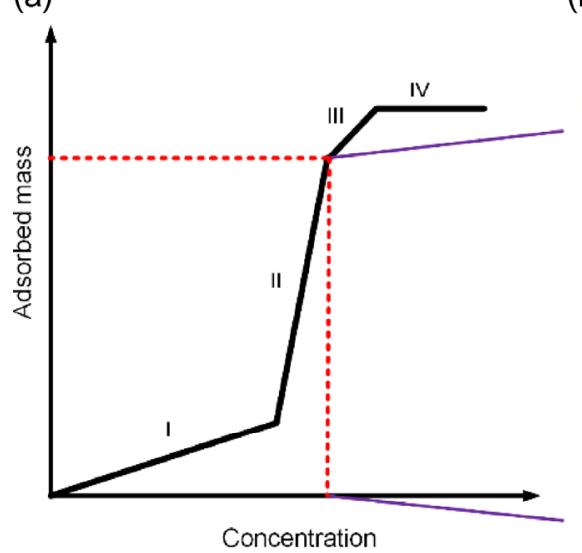

(b)
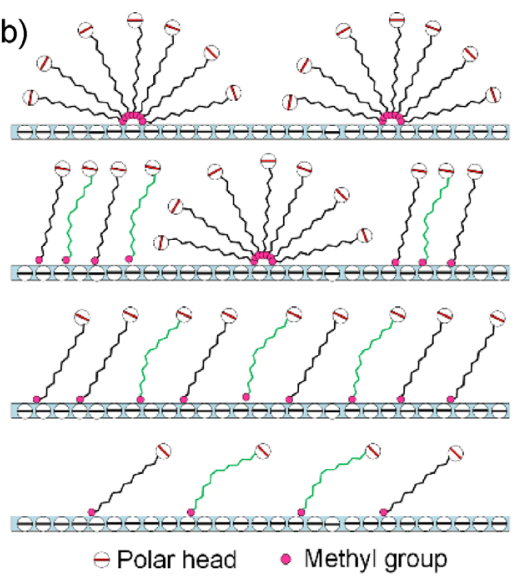

(c)

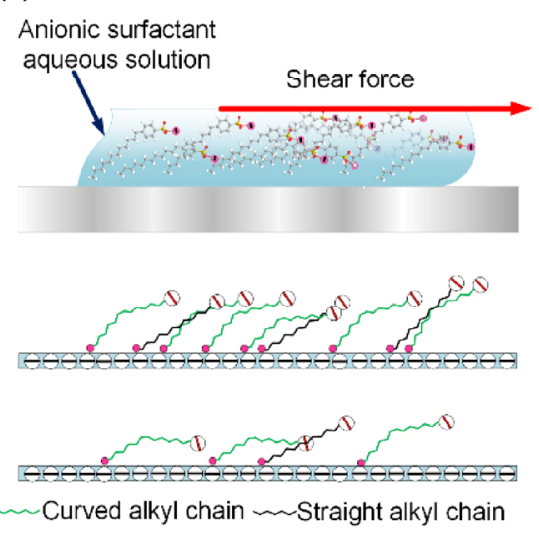

Fig. 4 (a) A general adsorption isotherm for the four-stage model; (b) the schematic diagram of four-stage adsorption model of SDBS on fused silica surface. The headgroups and alkyl chains of SDBS anion are represented by the circles with negative sign and curved lines, respectively; and (c) when the anionic surfactant aqueous solution layer is applied a shear stress, the molecular arrangement changes.

[22]. The anions are relatively loosely arranged with each other, and their structures are relative disordered. The tilt angle relative to the interface normal is around $30^{\circ}$ when the concentration is $0.01 \mathrm{mM}$. 2) When the bulk concentration reaches critical hemimicelle concentration (HMC), hemimicelles start to aggregate on substrate surface. Once it exceeds HMC, the hemimicelles on substrate surface will compact. As the concentration of the bulk structure increases, more and more anions start adsorbing on the interface, and the arrangement between the anions becomes tight. The monolayers gradually become more orderly, that is, the alkyl chain becomes straighter. Because the interaction between the alkyl chains becomes stronger, there is not enough space for the disordered chain. The resulting average tilt angle of alkyl chain becomes smaller with the increasing concentration. 3) Once the entire substrate surface is covered by the micellization, the formation of micelles starts in the bulk solution. 4) The number of micelles grow continuously when the solution reaches $\mathrm{CMC}$, and then achieve a relatively stable state.

In this study, we are mainly focused on the conformation order and tilt angle of anionic surfactant at low concentrations (at Stages I and II) under shear force. It is also an improvement and supplement of the adsorption model in Ref. [22]. The schematic is shown in Fig. 4(c), at the first two stages, when the anionic surfactant aqueous solution layer is applied a shear stress, the molecular arrangement changes. The long- chain molecules might be intertwined with each other. Therefore, the molecular conformation becomes more disordered due to friction. In addition, the tilt angle becomes larger, that is the angle between methyl and horizontal line turns to smaller. This occurs may mostly through the changes of interactions between chains, hydrophobic interaction, van der Waals forces, and electrostatic interaction. More further explorations for the underlying mechanisms are still needed.

\section{Conclusions}

The orientation angle and conformation order for molecular monolayers of SDBS at low concentrations on fused silica was studied by using SFG-VS. A modified four stage adsorption model considering friction effect was presented. At low concentrations, the alkyl chain of the anionic surfactant SDBS had a strong Gauche defect, indicating that the conformation of the anionic surfactant at the interface is relatively disordered. Meanwhile, the SDBS anion was arranged on the interface at a certain orientation angle due to the electrostatic repulsion. When the SDBS concentration increases, more molecules adsorbed on the surface. As a result, the Gauche defect decreased due to the chain-chain interaction at higher concentrations. As the concentration of SDBS increases, the molecular arrangement became well-ordered, and the orientation angle decreased. There existed a reorientation process of the alkyl chain during friction. The molecular 
arrangement was disordered, and orientation angle became larger due to friction. It is most likely a combination of several interactions involved such as the interaction between chains, hydrophobic interaction, van der Waals forces, and electrostatic interaction.

\section{Acknowledgements}

The work was financially supported by the National Natural Science Foundation of China (Nos. 51675297 and 51527901).

Electronic Supplementary Material: Supplementary material is available in the online version of this article at https://doi.org/10.1007/10.1007/s40544-021-0541-1.

Open Access This article is licensed under a Creative Commons Attribution 4.0 International License, which permits use, sharing, adaptation, distribution and reproduction in any medium or format, as long as you give appropriate credit to the original author(s) and the source, provide a link to the Creative Commons licence, and indicate if changes were made.

The images or other third party material in this article are included in the article's Creative Commons licence, unless indicated otherwise in a credit line to the material. If material is not included in the article's Creative Commons licence and your intended use is not permitted by statutory regulation or exceeds the permitted use, you will need to obtain permission directly from the copyright holder.

To view a copy of this licence, visit http://creativecommons.org/licenses/by/4.0/.

\section{References}

[1] Hore D K, Beaman D K, Richmond G L. Surfactant headgroup orientation at the air/water interface. J Am Chem Soc 127(26): 9356-9357 (2005)

[2] Burgess I, Zamlynny V, Szymanski G, Lipkowski J, Majewski J, Smith G, Satija S, Ivkov R. Electrochemical and neutron reflectivity characterization of dodecyl sulfate adsorption and aggregation at the gold-water interface. Langmuir 17(11): 3355-3367 (2001)

[3] Soares DM, Gomes WE, Tenan MA. Sodium dodecyl sulfate adsorbed monolayers on gold electrodes. Langmuir 23(8): 4383-4388 (2007)
[4] Manne S, Gaub H E. Molecular organization of surfactants at solid-liquid interfaces. Science 270(5241): 1480-1482 (1995)

[5] Kékicheff P, Contal C. Cationic-surfactant-coated mica surfaces below the critical micellar concentration: 1. patchy structures as revealed by peak force tapping AFM mode. Langmuir 35(8): 3087-3107 (2019)

[6] Wanless E J, Ducker W A. Organization of sodium dodecyl sulfate at the graphite-solution interface. J Phys Chem 100(8): 3207-3214 (1996)

[7] Schniepp H C, Saville D A, Aksay I A. Self-healing of surfactant surface micelles on millisecond time scales. $J$ Am Chem Soc 128(38): 12378-12379 (2006)

[8] Duan M, Wang H, Fang S W, Liang Y. Real-time monitoring the adsorption of sodium dodecyl sulfate on a hydrophobic surface using dual polarization interferometry. $J$ Colloid Interface Sci 417: 285-292 (2014)

[9] Das S K, Sengupta S, Velarde L. Interfacial surfactant ordering in thin films of SDS-encapsulated single-walled carbon nanotubes. J Phys Chem Lett 7(2): 320-326 (2016)

[10] Algoul ST, Sengupta S, Bui TT, Velarde L. Tuning the surface ordering of self-assembled ionic surfactants on semiconducting single-walled carbon nanotubes: Concentration, tube diameter, and counterions. Langmuir 34(31): 9279-9288 (2018)

[11] Song B, Xu P, Zeng G M, Gong J L, Wang X X, Yan J, Wang S F, Zhang P, Cao W C, Ye S J. Modeling the transport of sodium dodecyl benzene sulfonate in riverine sediment in the presence of multi-walled carbon nanotubes. Water Res 129: 20-28 (2018)

[12] Paria S, Manohar C, Khilar K C. Adsorption of anionic and non-ionic surfactants on a cellulosic surface. Colloids Surf A: Physicochem Eng Aspects 252(2-3): 221-229 (2005)

[13] Paria S, Manohar C, Khilar K C. Kinetics of adsorption of anionic, cationic, and nonionic surfactants. Ind Eng Chem Res 44(9): 3091-3098 (2005)

[14] Golub T P, Koopal L K, Sidorova M P. Adsorption of cationic surfactants on silica surface: 1 . adsorption isotherms and surface charge. Colloid J 66(1): 38-43 (2004)

[15] Tyrode E, Rutland M W, Bain C D. Adsorption of CTAB on hydrophilic silica studied by linear and nonlinear optical spectroscopy. J Am Chem Soc 130(51): 17434-17445 (2008)

[16] Gao Y Y, Du J Z, Gu T R. Hemimicelle formation of cationic surfactants at the silica gel-water interface. $J$ Chem Soc, Faraday Trans 1 83(8): 2671 (1987)

[17] Takeshita N, Okuno M, Ishibashi T A. Development of heterodyne-detected total internal reflection vibrational sum frequency generation spectroscopy and its application to $\mathrm{CaF}_{2}$ /liquid interfaces. J Phys Chem C 121(45): 2520625214 (2017) 
[18] Somasundaran P, Fuerstenau D W. Mechanisms of alkyl sulfonate adsorption at the alumina-water Interface1. J Phys Chem 70(1): 90-96 (1966)

[19] Pham T D, Kobayashi M, Adachi Y. Adsorption of anionic surfactant sodium dodecyl sulfate onto alpha alumina with small surface area. Colloid Polym Sci 293(1): 217-227 (2015)

[20] Ma K, Cui L Y, Dong Y Z, Wang T L, Da C, Hirasaki G J, Biswal S L. Adsorption of cationic and anionic surfactants on natural and synthetic carbonate materials. $J$ Colloid Interface Sci 408: 164-172 (2013)

[21] Hemmati N, Tabzar A, Ghazanfari M H. Adsorption of sodium dodecyl benzene sulfonate onto carbonate rock: Kinetics, equilibrium and mechanistic study. J Dispers Sci Technol 39(5): 687-699 (2018)

[22] Zhang J, Meng Y G, Tian Y, Zhang X J. Effect of concentration and addition of ions on the adsorption of sodium dodecyl sulfate on stainless steel surface in aqueous solutions. Colloids Surf A: Physicochem Eng Aspects 484: 408-415 (2015)

[23] Zhuang X, Miranda P B, Kim D, Shen Y R. Mapping molecular orientation and conformation at interfaces by surface nonlinear optics. Phys Rev B 59(19): 12632-12640 (1999)

[24] Meng Y G, Xu J, Jin Z M, Prakash B, Hu Y Z. A review of recent advances in tribology. Friction 8(2): 221-300 (2020)

[25] Ghaednia H, Wang X Z, Saha S, Xu Y, Sharma A, Jackson R L. A review of elastic-plastic contact mechanics. Appl Mech Rev 69(6): 060804 (2017)

[26] Ma L R, Luo J B. Thin film lubrication in the past 20 years. Friction 4(4): 280-302 (2016)

[27] Zhang S H, Qiao Y J, Liu Y H, Ma L R, Luo J B. Molecular behaviors in thin film lubrication-Part one: Film formation for different polarities of molecules. Friction 7(4): 372-387 (2019)

[28] Gao M, Li H Y, Ma L R, Gao Y, Ma L W, Luo J B. Molecular behaviors in thin film lubrication-Part two: Direct observation of the molecular orientation near the solid surface. Friction 7(5): 479-488 (2019)

[29] Ge X Y, Halmans T, Li J J, Luo J B. Molecular behaviors in thin film lubrication-Part three: Superlubricity attained by polar and nonpolar molecules. Friction 7(6): 625-636 (2019)

[30] Ta T D, Ta H D, Tieu K A, Tran B H. Impact of chosen force fields and applied load on thin film lubrication. Friction 9(5): 1259-1274 (2021)

[31] Ren Y L, Zhang L, Xie G X, Li Z B, Chen H, Gong H J, Xu W H, Guo D, Luo J B. A review on tribology of polymer composite coatings. Friction 9(3): 429-470 (2021)

[32] Martín-Alfonso J E, Martín-Alfonso M J, Valencia C, Cuberes M T. Rheological and tribological approaches as a tool for the development of sustainable lubricating greases based on nano-montmorillonite and castor oil. Friction 9(2): 415-428 (2021)

[33] Zhang X H, Xu Y, Jackson R L. A mixed lubrication analysis of a thrust bearing with fractal rough surfaces. In $P$ I Mech Eng J-J Eng 234(4): 608-621 (2019)

[34] Li J J, Zhang C H, Cheng P, Chen X C, Wang W Q, Luo J B. AFM studies on liquid superlubricity between silica surfaces achieved with surfactant micelles. Langmuir 32(22): 5593-5599 (2016)

[35] Li J J, Li J F, Jiang L, Chen X C, Luo J B. Cationic surfactant micelles lubricate graphitic surface in water. Langmuir 35(34): 11108-11113 (2019)

[36] He S Q, Meng Y G, Tian Y. Correlation between adsorption/desorption of surfactant and change in friction of stainless steel in aqueous solutions under different electrode potentials. Tribol Lett 41(3): 485-494 (2011)

[37] Zhang J, Meng Y G. Stick-slip friction of stainless steel in sodium dodecyl sulfate aqueous solution in the boundary lubrication regime. Tribol Lett 56(3): 543-552 (2014)

[38] Lis D, Backus E H G, Hunger J, Parekh S H, Bonn M. Liquid flow along a solid surface reversibly alters interfacial chemistry. Science 344(6188): 1138-1142 (2014)

[39] Gao Y, Ma L R, Liang Y, Li B H, Luo J B. Water molecules on the liquid superlubricity interfaces achieved by phosphoric acid solution. Biosurface Biotribology 4(3): 94-98 (2018)

[40] Wang J, Chen C Y, Buck S M, Chen Z. Molecular chemical structure on poly(methyl methacrylate) (PMMA) surface studied by sum frequency generation (SFG) vibrational spectroscopy. J Phys Chem B 105(48): 12118-12125 (2001)

[41] Tyrode E, Hedberg J. A comparative study of the CD and $\mathrm{CH}$ stretching spectral regions of typical surfactants systems using VSFS: Orientation analysis of the terminal $\mathrm{CH}_{3}$ and CD3 groups. $J$ Phys Chem C 116(1): 1080-1091 (2012)

[42] Weeraman C, Yatawara A K, Bordenyuk A N, Benderskii A V. Effect of nanoscale geometry on molecular conformation: Vibrational sum-frequency generation of alkanethiols on gold nanoparticles. J Am Chem Soc 128(44): 14244-14245 (2006)

[43] Zhang H, Li F J, Xiao Q B, Lin H Z. Conformation of capping ligands on nanoplates: Facet-edge-induced disorder and self-assembly-related ordering revealed by sum frequency generation spectroscopy. J Phys Chem Lett 6(12): 2170 $2176(2015)$

[44] Ma Y X, Hou J, Hao W Y, Liu J C, Meng L W, Lu Z. Influence of riboflavin on the oxidation kinetics of unsaturated fatty acids at the air/aqueous interface revealed by sum frequency generation vibrational spectroscopy. Phys Chem Chem Phys 20(25): 17199-17207 (2018) 
[45] Pan X C, Yang F Y, Chen S L, Zhu X F, Wang C Y. Cooperative effects of zwitterionic-ionic surfactant mixtures on the interfacial water structure revealed by sum frequency generation vibrational spectroscopy. Langmuir 34(18): 52735278 (2018)

[46] Tyrode E, Johnson C M, Rutland M W, Claesson P M. Structure and hydration of poly(ethylene oxide) surfactants at the air/liquid interface. A vibrational sum frequency spectroscopy study. J Phys Chem C 111(31): 11642-11652 (2007)

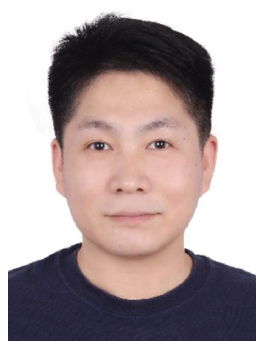

Xianzhang WANG. He received his M.S. and Ph.D. degrees in mechanical engineering from Auburn University in 2014 and 2017, respectively. Afterward, he worked as a postdoctoral research fellow working with Prof. Jianbin LUO

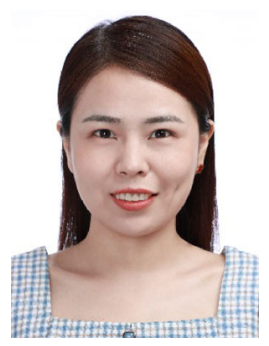

Yuan LIU. She received her B.S. degree in practical chemical engineering from Liaoning Petrochemical University in 2014, and got her M.S. degree from China University

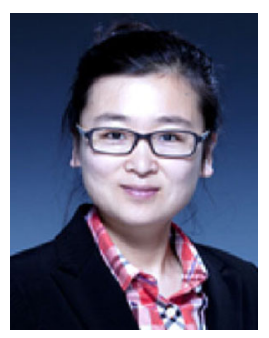

Liran MA. She received her B.S. and Ph.D. degrees from Tsinghua University in 2005 and 2010, respectively. Following a postdoctoral period at Weizmann Institute of Science in Israel, she is now working as an associate professor
[47] Schleeger M, Nagata Y, Bonn M. Quantifying surfactant alkyl chain orientation and conformational order from sum frequency generation spectra of $\mathrm{CH}$ modes at the surfactantwater interface. J Phys Chem Lett 5(21): 3737-3741 (2014)

[48] Velarde L, Zhang X Y, Lu Z, Joly A G, Wang Z M, Wang H F. Communication: Spectroscopic phase and lineshapes in high-resolution broadband sum frequency vibrational spectroscopy: Resolving interfacial inhomogeneities of “identical” molecular groups. J Chem Phys 135(24): 241102 (2011)

and Prof. Liran MA at the State Key Laboratory of Tribology, Tsinghua University, Beijing, China. He is currently a lecturer at Guangdong Ocean University, China. He has published more than 10 papers in international journals. His major research areas include orientation and structure of molecular, contact mechanics, and antifouling materials.

of Petroleum (Beijing) in 2018. She is currently perusing the Ph.D. degree from Tsinghua University. Her research interests are mainly about orientation and structure of molecular and sum frequency generation.

in the State Key Laboratory of Tribology, Tsinghua University. Her interests in tribology have ranged from aqueous lubrication and hydration lubrication to the liquid/solid interface properties. She has published over 80 papers. Her work has been cited more than 900 times. 